Case report:

\title{
Challenges During Modified Constraint Induced Movement Therapy (mCIMT) in Hemiplegic Cerebral Palsy \\ Mazatulfazura SF Salim ${ }^{1}$, Aneesa Abdul Rashid ${ }^{2}$, Muhammad Hibatullah Romli ${ }^{3}$
}

\begin{abstract}
:
Two cases involving children with hemiplegic cerebral palsy aged 1 and 8 year receiving mCIMT were reported. Challenges faced by the clinicians and family members were discussed in detail. mCIMT is rarely implemented in pediatric rehabilitation in Malaysia. The intensive protocol of mCIMT yielded challenges in terms of high commitment from the family members and non-adherence from the children. Clinicians and family members should properly plan and discuss when consideringmCIMT for intervention.
\end{abstract}

Keywords: Cerebral palsy, hemiplegia, pediatrics, modified constraint induced movement therapy.

International Journal of Human and Health Sciences Vol. 04 No. 03 July'20 Page : 229-231 DOI: http://dx.doi.org/10.31344/ijhhs.v4i3.206

\section{Case 1}

$\mathrm{SNI}$ is a 1 year and 3 months old girl, who was born premature at 27 weeks POA and was complicated with Respiratory Distress Syndrome that required intubation and NICU admission for almost a month. The child's mother noted that at 9 months old, she had developed right hand dominance and her left hand was kept fisting. Her mother also noted that at 1 year old, SNI was moving around with her bottom shuffling. She was unable to pull herself in order to stand and this was mainly limited by her left upper and lower limb weakness. Upon examination, she was not moving much of her left hand however able to open fingers occasionally. When standing, it was observed that she bears weight only at the right lower limb.

Besides the neurodevelopmental therapy (NDT) approach, we had discussed with the patient's mother regarding the benefit of mCIMT to improve her daughter's left upper limb function. The unaffected upper limb is restrained during therapy session for 2 hours per day, every day for two weeks at home. The home programme was provided by the clinicians for the caregivers to follow. Two one-hour therapy sessions every week at the clinic was conducted to monitor the intervention. Crepe bandage was used as restrainer. Intensive and repetitive practices of motor activities were incorporated. Activities such as reaching, grasping, holding, manipulating an object and bearing weight on the arm were introduced.

After 1 week of treatment, the parents had expressed difficulties to comply with the protocol at home as itconsumed their time. Furthermore, they noted that SNI was feeling uncomfortable with the restraint and at times, managed to remove it on her own. The parents were unable to proceed with the protocol due to the time factor and also, they felt uncomfortable when their child became more distressed and frustrated after being restrained.

\section{Case 2}

JOL is an 8 years old boy with underlying hypoplastic left heart. He underwent multiple surgeries at the age of 5 but developed thromboembolic complication post-surgery. Since then, he developed left hemiparesis. He had typicaldevelopmental milestone, attending normal school and able to participate in the normal learning and teaching session. He was able to ambulate independently without aids. $\mathrm{He}$ independently performed his personal activity of daily livings however a little slow in dressing

1. Department of Medicine, Faculty of Medicine and Health Sciences, UPM, 43400 Serdang, Malaysia.

2. Department of Family Medicine, Faculty of Medicine and Health Sciences, UPM, 43400 Serdang, Malaysia.

3. Department of Nursing and Rehabilitation, Faculty of Medicine and Health Sciences, UPM, 43400 Serdang, Malaysia.

Correspondence to: Mazatulfazura SF Salim, Department of Medicine, Faculty of Medicine and Health Sciences, UPM, 43400 Serdang, Malaysia. E-mail: fazurasf@upm.edu.my. 
and bathing. On examination, his left shoulder abduction and elbow extension/flexion were $4 / 5$, wrist extension/flexion were $3 / 5$ and his finger extension and flexion were $3 / 5$ with fair grip strength. He had mild spasticity over his biceps, FCU and FCR with Modified Ashworth Scale (MAS) of 1. His left lower limb proximal muscles were $4 / 5$ and distal muscles were $3 / 5$. There was only mild spasticity over his hamstring and gastrocsoleus with MAS of $1+$. He had hemiplegic gait with extensor synergy pattern with good ground clearance. He underwent physiotherapy for stretching and strengthening exercise.

mCIMT was suggested as an additional intervention. The mCIMT program was introduced to the patient whereby restraint of the unaffected upper limb is applied, and 2 hours per day of therapy is provided to the affected limb for 2 weeks whereby $2 \mathrm{x}$ per week ( 2 hours per week) sessions are with the therapist and at least 2 hours per day structured practice with the caregivers. The type of restraint for this patient is by also using a crepe bandage, and intensive, repetitive practices of motor activities were incorporated. Task such as reaching, grasping, holding, manipulating an object and bearing weight on the arm were introduced to the patient. These tasks were also taught to the parents as they are supposed to apply it daily to the patient. Interesting age-appropriate activities such as games, puzzle, 'blocks and hole' and kid's clay is provided. The mother of this patient is a housewife and she was able to spend time and focused on the intensive therapy needed while at home. However, being a bright kid, JOL had always found a way or giving excuses not to restraint his non paretic hand and refused to participate during the therapy. He will refuse to eat or bathe if he was restrained and was persuaded to use his paretic hand. Due to the patient's own noncompliance, the mCIMT was unable to be completed.

\section{Discussion}

CIMT and related interventions (mCIMT and Forced Use CIMT) are one of the promising interventions to reduce impairment and improve functional use of the affected upper limb of children with hemiplegia and cerebral palsy ${ }^{1}$. However, several factors need to be considered to ensure its efficacy. In this article, several factors have been identified on the unsuccessful in initiating mCIMT.

CIMT and its related interventions are relatively new and rarely implemented in Malaysia. Studies indicated that the protocol on restraining, and dosage is varied among literature and there is no standard accepted practice available ${ }^{1}$. Even in Western countries, the CIMT is criticized on its high-intensive protocol ${ }^{1}$. CIMT is originally developed in the Western countries and thus,its feasibility to be implemented in Southeast Asian especially in Malaysia is not explored. Culture, perception and lifestyle of Malaysian community such as long working hours of parents, pessimistic social perception towards disabilities and limited inclusion of children with disabilities into prime school settings may explain why poor adherence on CIMT intervention happen.

This case report indicates active involvement from the parents or caregivers on giving feedback about the intervention. Therefore, it is an opportunity to conduct rectifying measure to overcome the challenges to successfully carrying the mCIMT intervention rather than stopping the intervention. Clinicians have role to be equipped with knowledge on CIMT, develop a detail home programme. They should actively involve with the parents, family members, caregivers and the child in the discussion and planning the intervention goal and expectation ${ }^{2}$. Proper pre- and post-assessment should be performed to show the parents on the benefits of CIMT and constant explanation should be given to motivate the parents and the child to continue the intervention. Innovative approach on CIMT should be introduced. Rather than 2 hours continuous, the dosage could be break into several sessions with the cumulative time to be 2 hours per day. The CIMT is conducted when the child doing daily activities such as during dinner time (feeding activities using the affected hand) and during doing the homework. This may help the child to be more engage, increase attention and reduce the parents' commitment time.

The clinicians and therapists play a major role in implementing mCIMT. Guideline and Recommendation for CIMT by Cincinnati Children Hospital 2009, emphasized on the needs for the clinicians and therapists to have skills and knowledge in CIMT/mCIMT theory, evidence based practice, clinical guidelines, assessments, goal setting, and development of home programming materials. They also recommended that the clinicians and therapists to be able to give in-depth education to the caregivers prior to implementing CIMT to assist them in understanding the commitment necessary for successful completion of the CIMT program ${ }^{3}$. In our cases that we presented here, there was 
lack of assessment prior to initiating mCIMT and there were possibilities that the caregivers were not given in depth explanation on mCIMT and the importance or needs for compliancy to the structured home programming.

Patient factors also play a major role in ensuring efficacy of CIMT. There were several factors that might affect the compliancy towards the program. One of the factors is the method and fabrication of the constraint. Various constraints have been studied in the literature but there is insufficient evidence to support the use of a specific type ${ }^{4}$. Older children who are able to understand the reason for constraint use may be able to use less restrictive constraints such as ace wrap to the unaffected arm. However younger children might require a more robust constraint i.e. cast from which they cannot slip out ${ }^{1}$.

Some parents were concerned with the possible negative effects of mCIMT on the child. With the constraint used especially in children, lead to possibility of increased frustration which impacts the self-esteem. Studies of group therapy reported that parents and therapists observed that their children had higher self-esteem and increased motivation to participate in difficult activities. In group therapy, they also showed improvements in performance because they could model their actions after their peers, and they were more willing to persist in difficult or frustrating activities. Furthermore, they felt a sense of belonging, and appreciated seeing other children with similar motor deficits ${ }^{5,6}$. Our patients that we presented above might also benefit from a group therapy.

To conclude, there are needs of commitment and participation of children, their families, the clinicians and therapists during mCIMT in order to ensure efficacy of the treatments in this pediatric group of patients. Shared decision making and goal setting between caregivers, the clinicians and therapists are also paramount.

\section{Conflict of interest}

No conflict of interest has been disclosed by the authors.

\section{Ethical approval issue}

The authors acknowledged that consent has been obtained from the patients in regard to the details included in this report. Reporting of this study has been checked and verified in accordance with the CARE (Consensus based Clinical Case Reporting Guideline Development) checklist.

\section{Funding statement}

This study did not receive any funding.

\section{Authors Contributions}

Conception and design: MSS, MHR, AAR; Critical revision of the article for important intellectual content: MSS, AAR, MHR.

\section{References:}

1. Chiu HC, Ada L. Constraint-inducedmovement therapy improves upper limb activity and participationin hemiplegic cerebral palsy: asystematic review. Journal of Physiotherapy 2016;62(3):130-7.

2. AdamsJR, Drake RE, Shared decision-making and evidence-based practice. Community Ment Health J, 2006;42(1):87-105.

3. Pediatric modified Constraint Induced Movement Therapy (mCIMT/BIT) Team, Cincinnati Children'sHospital Medical Center: Evidence-based clinical care guideline Pediatric modified Constraint Induced Movement Therapy (mCIMT) plus Bimanual Training (BIT), http://www.cincinnatichildrens. org/svc/alpha/h/health-policy/evbased / Pediatric modified Constraint Induced Movement Therapy
(mCIMT) plus Bimanual Training (BIT).htm, Guideline 34, pages 1- 21, December, 2014.

4. HoareBJ, et al., Constraint-induced movement therapy in the treatment of the upper limb in children with hemiplegic cerebral palsy. Cochrane Database Syst Rev, 2007;(2):Cd004149.

5. EliassonAC, et al., Feasibility of a day-camp model of modified constraint-induced movement therapy with and without botulinum toxin A injection for children with hemiplegia. Phys Occup Ther Pediatr, 2009;29(3):311-33.

6. Kruijsen-Terpstra, AJ, et al., Parents' experiences with physical and occupational therapy for their young child with cerebral palsy: A mixed studies review. Child Care Health Dev, 2014;40(6):787-96. 